\title{
Subordination and Superordination Results for a Class of Analytic Multivalent Functions
}

\author{
S. P. Goyal, ${ }^{1}$ Pranay Goswami, $^{1}$ and H. Silverman ${ }^{2}$ \\ ${ }^{1}$ Department of Mathematics, University of Rajasthan, Jaipur 302004, India \\ ${ }^{2}$ Department of Mathematics, College of Charleston, Charleston, SC 29424, USA \\ Correspondence should be addressed to H. Silverman, silvermanh@cofc.edu \\ Received 25 September 2007; Revised 19 February 2008; Accepted 21 May 2008 \\ Recommended by Dorothy Wallace
}

We derive subordination and superordination results for a family of normalized analytic functions in the open unit disk defined by integral operators. We apply this to obtain sandwich results and generalizations of some known results.

Copyright (C) 2008 S. P. Goyal et al. This is an open access article distributed under the Creative Commons Attribution License, which permits unrestricted use, distribution, and reproduction in any medium, provided the original work is properly cited.

\section{Introduction}

Let $\mathscr{L}$ denote the class of analytic functions in the unit disk $\Delta:=\{z:|z|<1\}$, and let $\mathscr{L}[a, p]$ be the subclass of $\mathscr{t}$ of the form

$$
f(z)=a+a_{p} z^{p}+a_{p+1} z^{p+1}+\cdots, \quad p \in N=\{1,2,3, \ldots\} .
$$

Let $\mathcal{A}(p)$ be the subclass of $\mathscr{t}$ of the form

$$
f(z)=z^{p}+\sum_{k=p+1}^{\infty} a_{k} z^{k}, \quad p \in N
$$

If $f$ and $g$ are analytic and there exists a Schwarz function $w(z)$, analytic in $\Delta$ with

$$
w(0)=0, \quad|w(z)|<1, \quad z \in \Delta,
$$

such that $f(z)=g(w(z))$, then the function $f$ is called subordinate to $g$ and is denoted by

$$
f \prec g \quad \text { or } f(z) \prec g(z), \quad z \in \Delta .
$$


In particular, if the function $g$ is univalent in $\Delta$, the above subordination is equivalent to

$$
f(0)=g(0), \quad f(\Delta) \subset g(\Delta) .
$$

Suppose $h$ and $k$ are analytic functions in $\Delta$ and $\phi(r, s, t ; z): C^{3} \times \Delta \rightarrow C$. If $h$ and $\phi\left(h(z), z h^{\prime}(z), z^{2} h^{\prime \prime}(z) ; z\right)$ are univalent and if $h$ satisfies the second-order superordination

$$
k(z) \prec \phi\left(h(z), z h^{\prime}(z), z^{2} h^{\prime \prime}(z) ; z\right),
$$

then $h$ is a solution of the differential superordination (1.6). Note that if $f$ is subordinate to $g$, then $g$ is superordinate to $f$. An analytic function $q$ is called subordinant if $q<h$ forall $h$ satisfying (1.6). A univalent subordinant $\tilde{q}$ that satisfies $q<\tilde{q}$ for all subordinants $q$ of (1.6) is said to be the best subordinant. Miller and Mocanu [1] have obtained conditions on $k, q$, and $\phi$ for which the following implication holds:

$$
k(z) \prec \phi\left(h(z), z h^{\prime}(z), z^{2} h^{\prime \prime}(z) ; z\right) \Longrightarrow q(z) \prec h(z) .
$$

Ali et al. [2] have obtained sufficient conditions for certain normalized analytic functions $f(z)$ to satisfy

$$
q_{1}(z) \prec \frac{z f^{\prime}(z)}{f(z)} \prec q_{2}(z),
$$

where $q_{1}$ and $q_{2}$ are given univalent functions in $\Delta$ with $q_{1}(0)=1$ and $q_{2}(0)=1$.

Recently, Shanmugam et al. $[3,4]$ have also obtained sandwich results for certain classes of analytic functions. Further subordination results can be found in [5-8].

\section{Definitions and Preliminaries}

Definition 2.1. For $f(z) \in \mathcal{A}(p)$, Shams et al. [9] defined the following integral operator:

$$
\begin{aligned}
\supset^{\sigma} f(z) & =\frac{(p+1)^{\sigma}}{z \Gamma(\sigma)} \int_{0}^{z}\left(\log \frac{z}{t}\right)^{\sigma-1} f(t) d t \\
& =z^{p}+\sum_{n=p+1}^{\infty}\left(\frac{p+1}{n+1}\right)^{\sigma} a_{n} z^{n}, \quad \sigma>0 .
\end{aligned}
$$

For the operator, one easily gets

$$
z\left[\supset^{\sigma} f(z)\right]^{\prime}=(p+1) \supset^{\sigma-1} f(z)-\supset^{\sigma} f(z) .
$$

Also for $-1 \leq B<A \leq 1$ and $\lambda \geq 0$, Shams et al. [9] defined a class $\Omega_{p}^{\sigma}(A, B ; \lambda)$ of functions $f(z) \in \mathcal{A}(p)$, so that

$$
\frac{\lambda}{p}\left(\frac{\partial^{\sigma-1} f(z)}{z^{p}}\right)+\frac{p-\lambda}{p}\left(\frac{\partial^{\sigma} f(z)}{z^{p}}\right) \prec \frac{1+A z}{1+B z} .
$$

The family $\Omega_{p}^{\sigma}(A ; B ; \lambda)$ is a general family containing various new and known classes of analytic functions (see, e.g., $[10,11])$. 
Definition 2.2 (see [1]). Denote by $Q$ the set of all functions $f(z)$ that are analytic and injective on $\bar{\Delta}-E(f)$, where

$$
E(f)=\left\{\zeta \in \partial \Delta: \lim _{z \rightarrow \zeta} f(z)=\infty\right\},
$$

and are such that $f^{\prime}(\zeta) \neq 0$ for $\zeta \in \partial \Delta-E(f)$.

We will require certain results due to Miller and Mocanu [1, 12], Bulboacă [13], and Shanmugam et al. [4] contained in the following lemmas.

Lemma 2.3 (see [12]). Let $q(z)$ be univalent in the unit disk $\Delta$, and let $\theta$ and $\phi$ be analytic in the domain $D$ containing $q(\Delta)$ with $\phi(w) \neq 0$ when $w \in q(\Delta)$. Set $Q(z)=z q^{\prime}(z) \phi(q(z)), h(z)=$ $\theta(q(z))+Q(z)$. Suppose that

(i) $Q(z)$ is starlike univalent in $\Delta$;

(ii) $\operatorname{Re}\left(z h^{\prime}(z) / Q(z)\right)>0$ for $z \in \Delta$.

If $p(z)$ is analytic in $\Delta$, with $p(0)=q(0), p(\Delta) \in D$, and

$$
\theta(p(z))+z p^{\prime}(z) \phi(p(z)) \prec \theta(q(z))+z q^{\prime}(z) \phi(q(z)),
$$

then $p(z) \prec q(z)$ and $q(z)$ is the best dominant.

Lemma 2.4 (see [4]). Let $q(z)$ be a convex univalent function in $\Delta$ and $\psi, \gamma \in \mathcal{C}$ with $\operatorname{Re}(1+$ $\left.\left(z q^{\prime \prime}(z) / q^{\prime}(z)\right)\right)>\max \{0,-\operatorname{Re}(\psi / \gamma)\}$. If $p(z)$ is analytic in $\Delta$ and

$$
\psi p(z)+\gamma z p^{\prime}(z) \prec \psi q(z)+\gamma z q^{\prime}(z),
$$

then $p(z) \prec q(z)$ and $q(z)$ is the best dominant.

Lemma 2.5 (see [12]). Let $q(z)$ be univalent in $\Delta$, and let $\phi(z)$ be analytic in a domain containing $q(\Delta)$. If $z q^{\prime}(z) / \varphi(q(z))$ is starlike and

$$
z p^{\prime}(z) \varphi(p(z)) \prec z q^{\prime}(z) \varphi(q(z)),
$$

then $p(z) \prec q(z)$ and $q(z)$ is the best dominant.

Lemma 2.6 (see [13]). Let $q(z)$ be convex univalent in the unit disk $\Delta$, and let $\vartheta$ and $\varphi$ be analytic in a domain $D$ containing $q(\Delta)$. Suppose that

(i) $\operatorname{Re}\left[\vartheta^{\prime}(q(z)) / \varphi(q(z))\right]>0$ for $z \in \Delta$;

(ii) $z q^{\prime}(z) \varphi(q(z))$ is starlike univalent in $z \in \Delta$.

If $p(z) \in \mathscr{H}[q(0), 1] \cap Q$, with $p(\Delta) \subseteq D$, and if $\vartheta(p(z))+z p^{\prime}(z) \phi(p(z))$ is univalent in $\Delta$ and

$$
\vartheta(q(z))+z q^{\prime}(z) \varphi(q(z)) \prec \vartheta(p(z))+z p^{\prime}(z) \varphi(p(z)),
$$

then $q(z) \prec p(z)$ and $q(z)$ is the best subordinant.

Lemma 2.7 (see [1]). Let $q(z)$ be convex univalent in $\Delta$ and $\gamma \in \mathcal{C}$. Further assume that $\operatorname{Re}(\gamma)>0$. If $p(z) \in \mathscr{H}[q(0), 1] \cap Q$ and $p(z)+\gamma z p^{\prime}(z)$ is univalent in $\Delta$, then

$$
q(z)+\gamma z q^{\prime}(z) \prec p(z)+\gamma z p^{\prime}(z),
$$

which implies that $q(z)<p(z)$ and $q(z)$ is the best subordinant. 
The main object of this paper is to apply a method based on the differential subordination in order to derive several subordination results.

\section{Subordination for analytic functions}

Theorem 3.1. Let $q(z)$ be univalent in the unit disk $\Delta, \lambda \in \mathcal{C}$, and

$$
\operatorname{Re}\left(1+\frac{z q^{\prime \prime}(z)}{q^{\prime}(z)}\right)>\max \left\{0,-\operatorname{Re}\left(\frac{p(p+1)}{\lambda}\right)\right\}, \quad \lambda \neq 0(p \in \mathbf{N})
$$

If $f(z) \in \mathcal{A}(p)$ satisfies the subordination

$$
\frac{\lambda}{p}\left(\frac{\partial^{\sigma-1} f(z)}{z^{p}}\right)+\frac{p-\lambda}{p}\left(\frac{\partial^{\sigma} f(z)}{z^{p}}\right) \prec q(z)+\frac{\lambda z q^{\prime}(z)}{p(p+1)},
$$

where $J^{\sigma} f(z)$ is defined by (2.1), then

$$
\left(\frac{\supset^{\sigma} f(z)}{z^{p}}\right) \prec q(z)
$$

and $q(z)$ is the best dominant.

Proof. Consider

$$
h(z):=\left(\frac{\partial^{\sigma} f(z)}{z^{p}}\right)
$$

Differentiating (3.4) with respect to $z$ logarithmically, we get

$$
\frac{z h^{\prime}(z)}{h(z)}=\frac{z\left[\partial^{\sigma} f(z)\right]^{\prime}}{\left.\jmath^{\sigma} f(z)\right)}-p .
$$

Now, in view of (2.3), we obtain from (3.5) the following subordination:

$$
h(z)+\frac{\lambda z h^{\prime}(z)}{p(p+1)} \prec q(z)+\frac{\lambda z q^{\prime}(z)}{p(p+1)} .
$$

An application of Lemma 2.4, with $\gamma=\lambda / p(p+1)$ and $\psi=1$, leads to (3.3).

Taking $q(z)=(1+A z) /(1+B z)$ in Theorem 3.1, we arrive at the following.

Corollary 3.2. Let $-1 \leq B<A \leq 1$ and $\operatorname{Re}((1-B z) /(1+B z))>\max \{0,-\operatorname{Re}(p(p+$ 1) $/ \lambda)\}(\lambda \neq 0), p \in \mathbf{N}$. If $f \in \mathcal{A}(p)$ and

$$
\frac{\lambda}{p}\left(\frac{\supset^{\sigma-1} f(z)}{z^{p}}\right)+\frac{p-\lambda}{p}\left(\frac{\supset^{\sigma} f(z)}{z^{p}}\right) \prec \frac{1+A z}{1+B z}+\frac{\lambda}{p(p+1)} \frac{(A-B) z}{(1+B z)^{2}},
$$

then

$$
\frac{\vartheta^{\sigma} f(z)}{z^{p}} \prec \frac{1+A z}{1+B z}
$$

and $(1+A z) /(1+B z)$ is the best dominant. 
Putting $p=1$ and $q(z)=(1+z) /(1-z)$ in Theorem 3.1, we get the following corollary.

Corollary 3.3. Let $\operatorname{Re}((1+z) /(1-z))>\max \{0,-\operatorname{Re}(2 / \lambda)\}$ and $\lambda \neq 0$. If $f \in \mathcal{A}(1)$ and

$$
\frac{\jmath \supset^{\sigma-1} f(z)}{z}+\frac{(1-\lambda) \supset^{\sigma} f(z)}{z} \prec \frac{1+z}{1-z}+\frac{\lambda z}{(1-z)^{2}}
$$

then

$$
\frac{\rho^{\sigma} f(z)}{z} \prec \frac{1+z}{1-z}
$$

and $(1+z) /(1-z)$ is the best dominant.

Theorem 3.4. Let $q(z)$ be univalent in $\Delta$ and $0 \neq \gamma, \mu \in \mathcal{C}$, and $\alpha, \beta \in \mathcal{C}$ such that $\alpha+\beta \neq 0$. Let $f \in \mathcal{A}(p)$ and suppose that $q$ satisfies

$$
\operatorname{Re}\left\{1+\frac{z q^{\prime \prime}(z)}{q^{\prime}(z)}-\frac{z q^{\prime}(z)}{q(z)}\right\}>0 .
$$

If

$$
1+\gamma \mu\left[\frac{\alpha z\left[\supset^{\sigma-1} f(z)\right]^{\prime}+\beta z\left[\supset^{\sigma} f(z)\right]^{\prime}}{\alpha \supset^{\sigma-1} f(z)+\beta \supset^{\sigma} f(z)}-p\right] \prec 1+\gamma \frac{z q^{\prime}(z)}{q(z)}
$$

then

$$
\left[\frac{\alpha \supset^{\sigma-1} f(z)+\beta \supset^{\sigma} f(z)}{(\alpha+\beta) z^{p}}\right]^{\mu} \prec q(z)
$$

and $q(z)$ is the best dominant.

Proof. Let us consider a function $h(z)$ defined by

$$
h(z):=\left[\frac{\alpha \supset^{\sigma-1} f(z)+\beta \supset^{\sigma} f(z)}{(\alpha+\beta) z^{p}}\right]^{\mu}, \quad \mu \neq 0, \alpha+\beta \neq 0 .
$$

Now, differentiating (3.14) logarithmically, we get

$$
\frac{z h^{\prime}(z)}{h(z)}=\mu\left[\frac{\alpha z\left[\supset^{\sigma-1} f(z)\right]^{\prime}+\beta z\left[\supset^{\sigma} f(z)\right]^{\prime}}{\alpha \supset^{\sigma-1} f(z)+\beta \supset^{\sigma} f(z)}-p\right] .
$$

By setting

$$
\theta(w)=1, \quad \phi(w)=\frac{\gamma}{w},
$$

it can be easily observed that $\theta(w)$ is analytic in $\mathcal{C}$ and that $\phi(w) \neq 0$ is analytic in $\mathcal{C} /\{0\}$. Also, we let

$$
\begin{gathered}
Q(z)=z q^{\prime}(z) \phi(q(z))=r \frac{z q^{\prime}(z)}{q(z)}, \\
p(z)=\theta(q(z))+Q(z)=1+\gamma \frac{z q^{\prime}(z)}{q(z)} .
\end{gathered}
$$


From (3.11) we see that $Q(z)$ is starlike univalent in the unit disk $\Delta$, and from (3.18) we get

$$
\operatorname{Re}\left(\frac{z p^{\prime}(z)}{Q(z)}\right)=\operatorname{Re}\left\{1+\frac{z q^{\prime \prime}(z)}{q^{\prime}(z)}-\frac{z q^{\prime}(z)}{q(z)}\right\}>0 .
$$

An application of Lemma 2.3 to (3.12) yields the result.

Putting $\alpha=0, \beta=1, \gamma=1$, and $q(z)=(1+A z) /(1+B z)$ in Theorem 3.4 , we obtain the following corollary.

Corollary 3.5. If $f(z) \in \mathcal{A}(p)$ and for $-1 \leq A<B \leq 1, \mu \neq 0$,

$$
1+\mu\left[\frac{z\left[\partial^{\sigma} f(z)\right]^{\prime}}{\partial^{\sigma} f(z)}-p\right] \prec 1+\frac{(A-B) z}{(1+A z)(1+B z)}
$$

then

$$
\left[\frac{\partial^{\sigma} f(z)}{z^{p}}\right]^{\mu} \prec \frac{1+A z}{1+B z}
$$

and $(1+A z) /(1+B z)$ is the best dominant.

By setting $\alpha=0, \beta=1, \gamma=1, \sigma=0, p=1$, and $q(z)=(1+B z)^{\mu(A-B) / B}$ inTheorem 3.4, we get the following corollary.

Corollary 3.6. Suppose $f(z) \in \mathcal{A}(1)$ and let $-1 \leq B<A \leq 1$ and $B \neq 0$. If

$$
1+\mu\left[\frac{z f^{\prime}(z)}{f(z)}-1\right] \prec \frac{1+A z}{1+B z}
$$

then

$$
\left[\frac{f(z)}{z}\right]^{\mu} \prec(1+B z)^{\mu(A-B) / B}
$$

and $(1+B z)^{\mu(A-B) / B}$ is the best dominant.

Remark 3.7. $q(z)=(1+B z)^{\mu(A-B) / B}$ is univalent if and only if $|(\mu(A-B) / B)-1| \leq 1$ or $\mid(\mu(A-$ $B) / B)+1 \mid \leq 1($ see $[5])$.

Again by setting $\beta=1, \mu=1, \alpha=0, \gamma=1 / b, p=1$, and $\sigma=0$, and by $q(z)=1 /(1-$ $z)^{2 b}(b \in \mathcal{C} \backslash\{0\})$ in Theorm 3.4, we get the following corollary.

Corollary 3.8. Suppose $f(z) \in \mathcal{A}(1)$ and $b$ is a nonzero complex number for which

$$
1+\frac{1}{b}\left[\frac{z f^{\prime}(z)}{f(z)}-1\right] \prec \frac{1+z}{1-z}
$$

Then,

$$
\frac{f(z)}{z} \prec \frac{1}{(1-z)^{2 b}}
$$

and $1 /(1-z)^{2 b}$ is the best dominant. 
The result contained in Corollary 3.8 was earlier given by Srivastava and Lashin [7].

Theorem 3.9. Let $q$ be univalent in the unit disk $\Delta$, and let $\mu, \gamma \neq 0, \eta, \delta, \alpha, \beta \in \mathcal{C}$, and $f(z) \in \mathcal{A}(p)$. Suppose that $q$ satisfies

$$
\operatorname{Re}\left\{1+\frac{z q^{\prime \prime}(z)}{q^{\prime}(z)}\right\}>\max \left\{0,-\operatorname{Re}\left(\frac{\eta}{r}\right)\right\}
$$

Let

$$
\psi(z)=\left[\frac{\alpha \supset^{\sigma-1} f(z)+\beta \supset^{\sigma} f(z)}{(\alpha+\beta) z^{p}}\right]^{\mu}\left\{\eta+\gamma \mu\left(\frac{\alpha z\left[\supset^{\sigma-1} f(z)\right]^{\prime}+\beta z\left[\supset^{\sigma} f(z)\right]^{\prime}}{\alpha \supset^{\sigma-1} f(z)+\beta \supset^{\sigma} f(z)}-p\right)\right\}+\delta .
$$

If

$$
\psi(z) \prec \eta q(z)+\delta+\gamma z q^{\prime}(z)
$$

then

$$
\left[\frac{\alpha \supset^{\sigma-1} f(z)+\beta \supset^{\sigma} f(z)}{(\alpha+\beta) z^{p}}\right]^{\mu} \prec q(z), \quad \alpha+\beta \neq 0,
$$

and $q(z)$ is the best dominant.

Proof. Define a function $h(z)$ by

$$
h(z):=\left[\frac{\alpha \supset^{\sigma-1} f(z)+\beta \supset^{\sigma} f(z)}{(\alpha+\beta) z^{p}}\right]^{\mu} .
$$

Then, a computation shows that

$$
\frac{z h^{\prime}(z)}{h(z)}=\mu\left\{\frac{\alpha z\left[\supset^{\sigma-1} f(z)\right]^{\prime}+\beta z\left[\supset^{\sigma} f(z)\right]^{\prime}}{\alpha \supset^{\sigma-1} f(z)+\beta \supset^{\sigma} f(z)}-p\right\}
$$

and hence

$$
z h^{\prime}(z)=\mu h(z)\left(\frac{z\left[\alpha \supset^{\sigma-1} f(z)\right]^{\prime}+z \beta\left[\supset^{\sigma} f(z)\right]^{\prime}}{\alpha \supset^{\sigma-1} f(z)+\beta \supset^{\sigma} f(z)}-p\right) .
$$

Set

$$
\theta(w)=\eta w+\delta, \quad \phi(w)=r
$$

and let

$$
\begin{gathered}
Q(z)=z q^{\prime}(z) \phi(q(z))=\gamma z q^{\prime}(z), \\
p(z)=\theta(q(z))+Q(z)=\eta q(z)+\delta+\gamma z q^{\prime}(z) .
\end{gathered}
$$

From (3.26), we see that $Q(z)$ is starlike in $\Delta$ and that

$$
\operatorname{Re}\left\{\frac{z p^{\prime}(z)}{Q(z)}\right\}=\operatorname{Re}\left\{\frac{\eta}{\gamma}+1+\frac{z q^{\prime \prime}(z)}{q^{\prime}(z)}\right\}>0,
$$

by the hypothesis (3.26) of Theorem 3.9. Thus, applying Lemma 2.3, the proof of Theorem 3.9 is completed. 

corollary.

By setting $\beta=1, \gamma=1, \alpha=0$, and $q(z)=(1+A z) /(1+B z)$, we obtain the following

Corollary 3.10. Let $f(z) \in \mathcal{A}(p)$ and $\operatorname{Re}(\eta)>0$. Suppose that

$$
\operatorname{Re}\left\{\frac{1-B z}{1+B z}\right\}>\max \{0,-\operatorname{Re}(\eta)\} \text {. }
$$

If

$$
\left[\frac{\partial^{\sigma} f(z)}{z^{p}}\right]^{\mu}\left\{\eta+\mu\left(\frac{z\left[\partial^{\sigma} f(z)\right]^{\prime}}{\partial^{\sigma} f(z)}-p\right)\right\}+\delta \prec \eta \frac{1+A z}{1+B z}+\delta+z \frac{(A-B)}{(1+B z)^{2}}
$$

then

$$
\left[\frac{\partial^{\sigma} f(z)}{z^{p}}\right]^{\mu} \prec \frac{1+A z}{1+B z}
$$

and $(1+A z) /(1+B z)$ is the best dominant.

Again by setting $\beta=1, \gamma=1, \alpha=0, p=1$, and $\sigma=0$, and by $q(z)=(1+z) /(1-z)$, we get the following corollary.

Corollary 3.11. Let $f(z) \in \mathcal{A}(1)$ and

$$
\left[\frac{f(z)}{z}\right]^{\mu}\left\{\eta+\mu\left(\frac{z f^{\prime}(z)}{f(z)}-1\right)\right\}+\delta \prec \eta \frac{1+z}{1-z}+\delta+\frac{2 z}{(1-z)^{2}},
$$

then

$$
\left[\frac{f(z)}{z}\right]^{\mu} \prec \frac{1+z}{1-z}
$$

and $(1+z) /(1-z)$ is the best dominant.

\section{Superordination for analytic functions}

Theorem 4.1. Let $q$ be convex univalent in the unit disk $\Delta$, and $\lambda \in \mathcal{C}$. Suppose $\lambda$ satisfies $\operatorname{Re}\{\lambda\}>0$ and $\supset^{\sigma} f(z) / z^{p} \in \mathscr{H}(q(0), 1) \cap Q$. Suppose that

$$
\frac{\lambda}{p}\left(\frac{\partial^{\sigma-1} f(z)}{z^{p}}\right)+\frac{p-\lambda}{p}\left(\frac{\partial^{\sigma} f(z)}{z^{p}}\right)
$$

is univalent in the unit disk $\Delta$. If

$$
q(z)+\frac{\lambda z q^{\prime}(z)}{p(p+1)} \prec \frac{\lambda}{p}\left(\frac{\partial^{\sigma-1} f(z)}{z^{p}}\right)+\frac{p-\lambda}{p}\left(\frac{\partial^{\sigma} f(z)}{z^{p}}\right),
$$

then

$$
q(z) \prec \frac{\partial^{\sigma} f(z)}{z^{p}}
$$

and $q(z)$ is the best subordinant. 
Proof. Let

$$
p(z)=\frac{\supset^{\sigma} f(z)}{z^{p}}, \quad z \neq 0
$$

Differentiating logarithmically, we get

$$
\frac{z p^{\prime}(z)}{p(z)}=\frac{z\left[\supset^{\sigma} f(z)\right]^{\prime}}{\supset^{\sigma} f(z)}-p .
$$

After some computation, we get

$$
p(z)+\frac{\lambda z p^{\prime}(z)}{p(p+1)}=\frac{\lambda}{p}\left(\frac{\partial^{\sigma-1} f(z)}{z^{p}}\right)+\frac{p-\lambda}{p}\left(\frac{\partial^{\sigma} f(z)}{z^{p}}\right) .
$$

Now, using Lemma 2.7, we get the desired result (4.3).

Corollary 4.2. Let $q$ be convex univalent in $\Delta$, and $\lambda \in C$. Suppose $\lambda$ satisfies $R\{\lambda\}>0$ and $\supset^{\sigma} f(z) / z^{p} \in \mathscr{L}(q(0), 1) \cap Q$. Let

$$
\frac{\lambda}{p}\left(\frac{\partial^{\sigma-1} f(z)}{z^{p}}\right)+\frac{p-\lambda}{p}\left(\frac{\partial^{\sigma} f(z)}{z^{p}}\right)
$$

be univalent in the unit disk $\Delta$. If

$$
\frac{\lambda(A-B) z}{p(p+1)(1+B z)^{2}}+\frac{1+A z}{1+B z} \prec \frac{\lambda}{p}\left(\frac{\supset^{\sigma-1} f(z)}{z^{p}}\right)+\frac{p-\lambda}{p}\left(\frac{\supset^{\sigma} f(z)}{z^{p}}\right),
$$

then

$$
\frac{1+A z}{1+B z} \prec \frac{\partial^{\sigma} f(z)}{z^{p}}
$$

and $(1+A z) /(1+B z)$ is the best subordinant.

Since the proofs of Theorems 4.3 and 4.4 are similar to the proofs of the previous theorems, we only give statements of these theorems without proofs.

Theorem 4.3. Let $q(z)$ be convex univalent in $\Delta$, and $0 \neq \gamma, \mu \in \mathcal{C}$, and $\alpha, \beta \in \mathcal{C}$ such that $\alpha+\beta \neq 0$. Let $f(z) \in \mathcal{A}(p)$. Suppose that $\left[\left(\alpha \supset^{\sigma-1} f(z)+\beta \supset^{\sigma} f(z)\right) /(\alpha+\beta) z^{p}\right]^{\mu} \in \mathcal{H}(q(0), 1) \cap Q$, and

$$
1+\gamma \mu\left[\frac{\alpha z\left[\supset^{\sigma-1} f(z)\right]^{\prime}+\beta z\left[\supset^{\sigma} f(z)\right]^{\prime}}{\alpha \supset^{\sigma-1} f(z)+\beta \supset^{\sigma} f(z)}-p\right]
$$

is univalent in $\Delta$. If

$$
1+\gamma \frac{z q^{\prime}(z)}{q(z)} \prec 1+\gamma \mu\left[\frac{\alpha z\left[\supset^{\sigma-1} f(z)\right]^{\prime}+\beta\left[\supset^{\sigma} f(z)\right]^{\prime}}{\alpha \supset^{\sigma-1} f(z)+\beta \supset^{\sigma} f(z)}-p\right]
$$

then

$$
q(z) \prec\left[\frac{\alpha \supset^{\sigma-1} f(z)+\beta \supset^{\sigma} f(z)}{(\alpha+\beta) z^{p}}\right]^{\mu}
$$

and $q(z)$ is the best subordinant. 
Theorem 4.4. Let $q$ be convex univalent in the unit disk $\Delta$, and let $\gamma \neq 0 \in \mathcal{C}, \eta, \delta, \alpha, \beta \in \mathcal{C}$ with $\alpha+\beta \neq 0$, and $f(z) \in \mathcal{A}(p)$. Suppose that $\supset^{\sigma} f(z) / z^{p} \in \mathscr{H}(q(0), 1) \cap Q$, and

$$
\operatorname{Re}\left\{\frac{\eta q^{\prime}(z)}{r}\right\}>0
$$

If

$\eta q(z)+\delta+\gamma z q^{\prime}(z) \prec\left[\frac{\alpha \supset^{\sigma-1} f(z)+\beta \supset^{\sigma} f(z)}{(\alpha+\beta) z^{p}}\right]^{\mu}\left\{\eta+\gamma \mu\left(\frac{z \alpha\left[\supset^{\sigma-1} f(z)\right]^{\prime}+z \beta\left[\supset^{\sigma} f(z)\right]^{\prime}}{\alpha \supset^{\sigma-1} f(z)+\beta \supset^{\sigma} f(z)}-p\right)\right\}+\delta$,

then

$$
q(z) \prec\left[\frac{\alpha \supset^{\sigma-1} f(z)+\beta \supset^{\sigma} f(z)}{(\alpha+\beta) z^{p}}\right]^{\mu}
$$

and $q(z)$ is the best subordinant.

\section{Sandwich results}

Combining results of differential subordinations and superordinations, we arrive at the following "sandwich results."

Theorem 5.1. Let $q_{1}(z)$ be convex univalent, and let $q_{2}(z)$ be univalent in $\Delta$, and $\lambda \in \mathcal{C}$. Suppose $q_{1}$ satisfies $\operatorname{Re}\{\lambda\}>0$ and $q_{2}$ satisfies (3.1). If $\supset^{\sigma} f(z) / z^{p} \in \mathscr{H}(q(0), 1) \cap Q$ and

$$
\left[\frac{\alpha \supset^{\sigma-1} f(z)+\beta \supset^{\sigma} f(z)}{(\alpha+\beta) z^{p}}\right]^{\mu}, \quad \alpha+\beta \neq 0
$$

is univalent in $\Delta$, and if

$$
q_{1}(z)+\frac{\lambda z q_{1}^{\prime}(z)}{p(p+1)} \prec \frac{\lambda \supset^{\sigma-1} f(z)}{z^{p}}+\frac{(p-\lambda) \supset^{\sigma} f(z)}{z^{p}} \prec q_{2}(z)+\frac{\lambda z q_{2}^{\prime}(z)}{p(p+1)},
$$

then

$$
q_{1}(z) \prec\left(\frac{\partial^{\sigma} f(z)}{z^{p}}\right) \prec q_{2}(z)
$$

and $q_{1}(z)$ and $q_{2}(z)$ are, respectively, the best subordinant and the best dominant.

Theorem 5.2. Let $q_{1}(z)$ be convex univalent, and let $q_{2}(z)$ be univalent in $\Delta$, and $\lambda \in \mathcal{C}$. Suppose that $q_{2}$ satisfies (3.11). Further suppose that $\left[\left(\alpha \supset^{\sigma-1} f(z)+\beta \supset^{\sigma} f(z)\right) /(\alpha+\beta) z^{p}\right]^{\mu} \in \mathscr{\ell}(q(0), 1) \cap Q$ and $1+\gamma \mu\left[\left(\alpha z\left[\supset^{\sigma-1} f(z)\right]^{\prime}+\beta z\left[\supset^{\sigma} f(z)\right]^{\prime}\right) /\left(\alpha \supset^{\sigma-1} f(z)+\beta \supset^{\sigma} f(z)\right)-p\right]$ is univalent in $\Delta$.

If

$$
1+\gamma \frac{z q_{1}^{\prime}(z)}{q_{1}(z)} \prec 1+\gamma \mu\left[\frac{\alpha z\left[\supset^{\sigma-1} f(z)\right]^{\prime}+\beta z\left[\supset^{\sigma} f(z)\right]^{\prime}}{\alpha \supset^{\sigma-1} f(z)+\beta \supset^{\sigma} f(z)}-p\right] \prec 1+\gamma \frac{z q_{2}^{\prime}(z)}{q_{2}(z)}
$$

then

$$
q_{1}(z) \prec\left[\frac{\alpha \supset^{\sigma-1} f(z)+\beta \supset^{\sigma} f(z)}{(\alpha+\beta) z^{p}}\right]^{\mu} \prec q_{2}(z), \quad \alpha+\beta \neq 0,
$$

and $q_{1}(z)$ and $q_{2}(z)$ are, respectively, the best subordinant and the best dominant. 
Theorem 5.3. Let $q_{1}(z)$ be convex univalent, and let $q_{2}(z)$ be univalent in $\Delta$, and $\lambda \in \mathcal{C}$. Suppose that $q_{1}(z)$ satisfies (4.13) and $q_{2}(z)$ satisfies (3.28). Further suppose that $\left[\left(\alpha \supset^{\sigma-1} f(z)+\right.\right.$ $\left.\left.\beta \supset^{\sigma} f(z)\right) /(\alpha+\beta) z^{p}\right]^{\mu} \in \mathscr{H}(q(0), 1) \cap Q$ with $\alpha+\beta \neq 0$, and that

$$
\left[\frac{\alpha \supset^{\sigma-1} f(z)+\beta \supset^{\sigma} f(z)}{(\alpha+\beta) z^{p}}\right]^{\mu}\left\{\eta+\gamma \mu\left(\frac{z \alpha\left[\supset^{\sigma-1} f(z)\right]^{\prime}+z \beta\left[\supset^{\sigma} f(z)\right]^{\prime}}{\alpha \supset^{\sigma-1} f(z)+\beta \supset^{\sigma} f(z)}-p\right)\right\}+\delta
$$

is univalent in $\Delta$. If

$$
\begin{aligned}
\eta q_{1}(z)+\delta+\gamma z q_{1}^{\prime}(z) & \prec\left[\frac{\alpha \supset^{\sigma-1} f(z)+\beta \supset^{\sigma} f(z)}{(\alpha+\beta) z^{p}}\right]^{\mu}\left\{\eta+\gamma \mu\left(\frac{z \alpha\left[\supset^{\sigma-1} f(z)\right]^{\prime}+z \beta\left[\supset^{\sigma} f(z)\right]^{\prime}}{\alpha \supset^{\sigma-1} f(z)+\beta \supset^{\sigma} f(z)}-p\right)\right\}+\delta \\
& \prec \eta q_{2}(z)+\delta+\gamma z q_{2}^{\prime}(z),
\end{aligned}
$$

then

$$
q_{1}(z) \prec\left[\frac{\alpha \supset^{\sigma-1} f(z)+\beta \supset^{\sigma} f(z)}{(\alpha+\beta) z^{p}}\right]^{\mu} \prec q_{2}(z)
$$

and $q_{1}(z)$ and $q_{2}(z)$ are, respectively, the best subordinant and the best dominant.

\section{Acknowledgments}

The authors are thankful to the referee for his useful suggestions. P. Goswami is also thankful to CSIR, India, for providing the Junior Research Fellowship under Research Scheme no. 09/135(0434)/2006-EMR-1.

\section{References}

[1] S. S. Miller and P. T. Mocanu, "Subordinants of differential superordinations," Complex Variables, vol. 48, no. 10, pp. 815-826, 2003.

[2] R. M. Ali, V. Ravichandran, M. H. Khan, and K. G. Subramanian, "Differential sandwich theorems for certain analytic functions," Far East Journal of Mathematical Sciences, vol. 15, no. 1, pp. 87-94, 2004.

[3] T. N. Shanmugam, S. Sivasubramanian, and H. Silverman, "On sandwich theorems for some classes of analytic functions," International Journal of Mathematics and Mathematical Sciences, vol. 2006, Article ID 29684, 13 pages, 2006.

[4] T. N. Shanmugam, V. Ravichandran, and S. Sivasubramanian, "Differential sandwich theorems for some subclasses of analytic functions," The Australian Journal of Mathematical Analysis and Applications, vol. 3, no. 1, article 8, pp. 1-11, 2006.

[5] M. Obradović and S. Owa, "On certain properties for some classes of starlike functions," Journal of Mathematical Analysis and Applications, vol. 145, no. 2, pp. 357-364, 1990.

[6] V. Singh, "On some criteria for univalence and starlikeness," Indian Journal of Pure and Applied Mathematics, vol. 34, no. 4, pp. 569-577, 2003.

[7] H. M. Srivastava and A. Y. Lashin, "Some applications of the Briot-Bouquet differential subordination," Journal of Inequalities in Pure and Applied Mathematics, vol. 6, no. 2, article 41, pp. 1-7, 2005.

[8] Z. Wang, C. Gao, and M. Liao, "On certain generalized class of non-Bazilevič functions," Acta Mathematica Academiae Paedagogicae Nyíregyháziensis, vol. 21, no. 2, pp. 147-154, 2005.

[9] S. Shams, S. R. Kulkarni, and J. M. Jahangiri, "Subordination properties of $p$-valent functions defined by integral operators," International Journal of Mathematics and Mathematical Sciences, vol. 2006, Article ID 94572, 3 pages, 2006. 
[10] J.-L. Liu, "Notes on Jung-Kim-Srivastava integral operator," Journal of Mathematical Analysis and Applications, vol. 294, no. 1, pp. 96-103, 2004.

[11] J. Patel and A. K. Mohanty, "On a class of $p$-valent analytic functions with complex order," Kyungpook Mathematical Journal, vol. 43, no. 2, pp. 199-209, 2003.

[12] S. S. Miller and P. T. Mocanu, Differential Subordinations: Theory and Applications, vol. 225 of Monographs and Textbooks in Pure and Applied Mathematics, Marcel Dekker, New York, NY, USA, 2000.

[13] T. Bulboacă, "Classes of first-order differential superordinations," Demonstratio Mathematica, vol. 35, no. 2, pp. 287-292, 2002. 


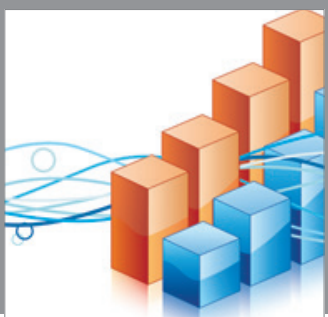

Advances in

Operations Research

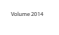

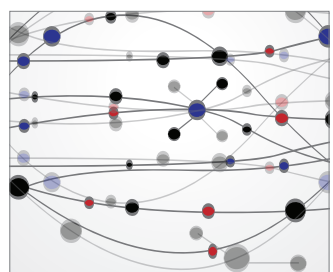

\section{The Scientific} World Journal
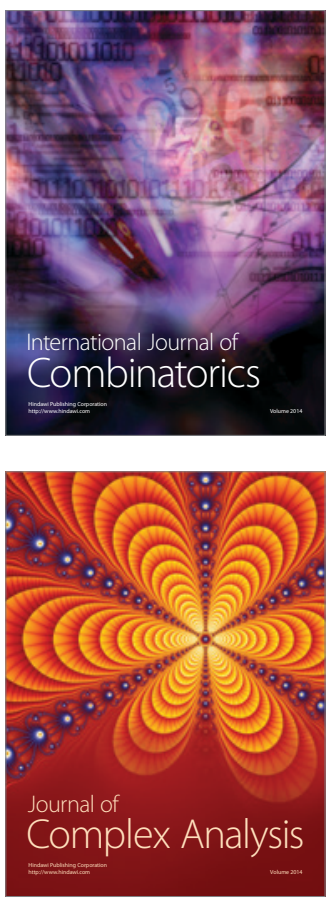

International Journal of

Mathematics and

Mathematical

Sciences
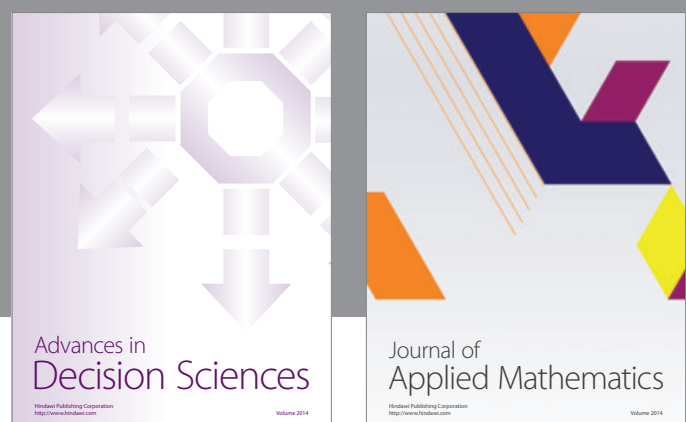

Journal of

Applied Mathematics
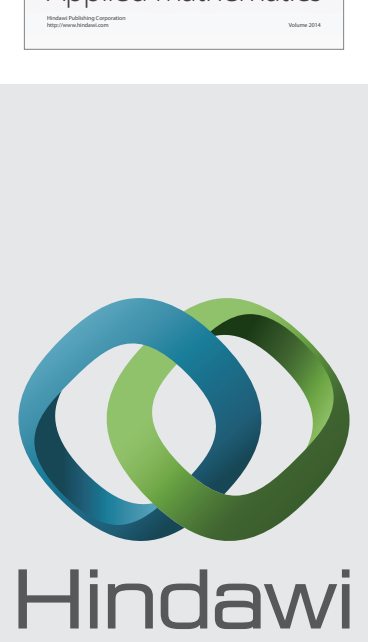

Submit your manuscripts at http://www.hindawi.com
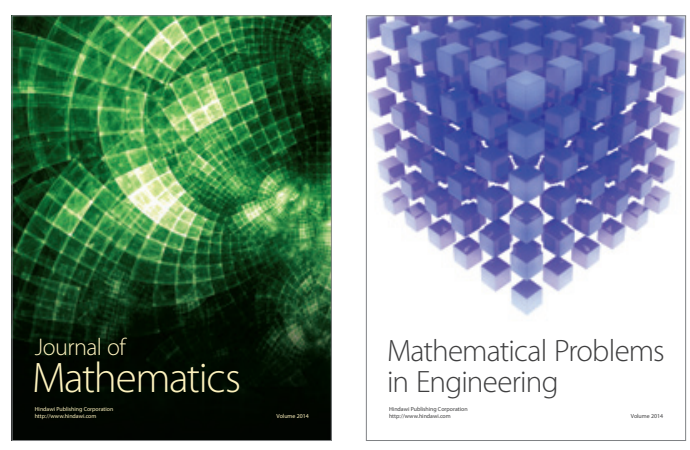

Mathematical Problems in Engineering
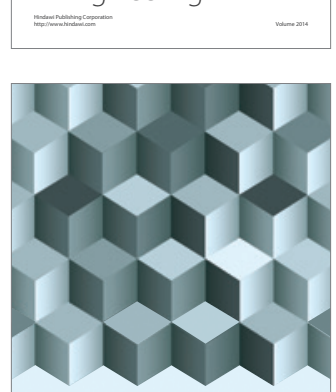

Journal of

Function Spaces
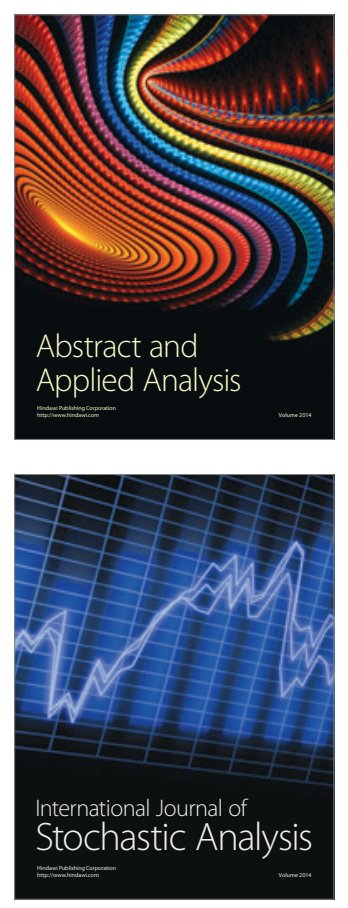

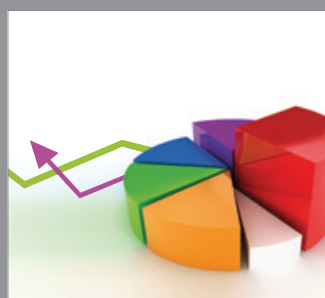

ournal of

Probability and Statistics

Promensencen
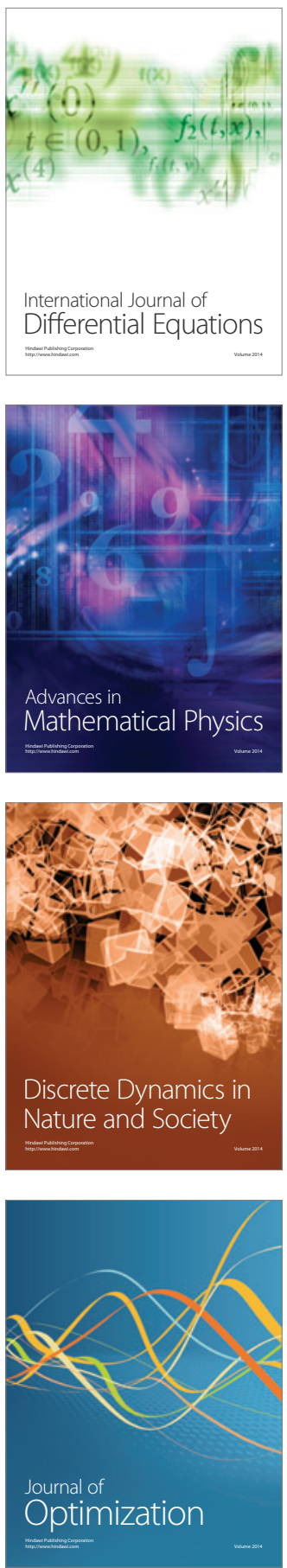